
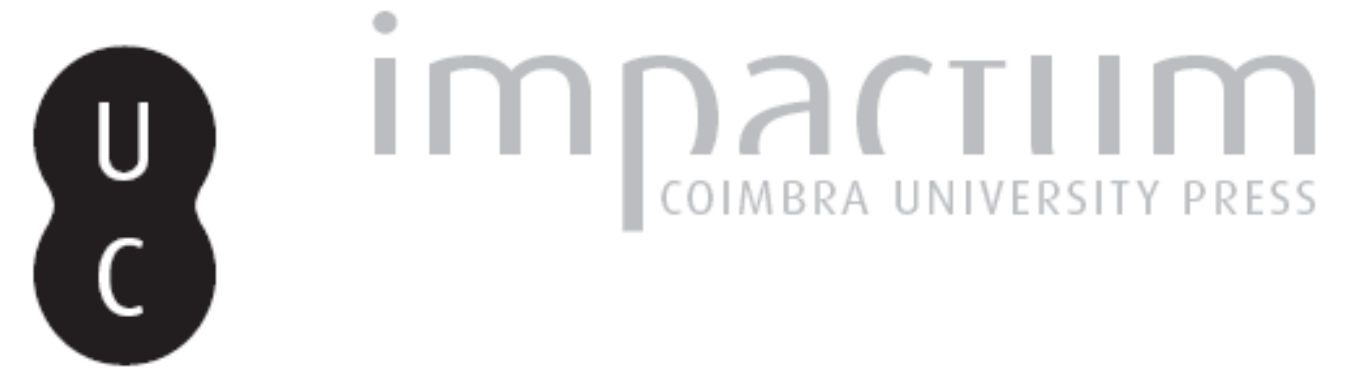

\title{
Archeologia della navigazione islamica d'occidente: lo studio di caso del Gharb portoghese
}

\author{
Autor(es): $\quad$ Amato, Alessia
}

Publicado por: Faculdade de Letras da Universidade de Coimbra

URL

persistente:

URI:http://hdl.handle.net/10316.2/38801

DOI:

DOI:http://dx.doi.org/10.14195/1647-8657_53_7

Accessed : $\quad$ 26-Apr-2023 14:13:32

A navegação consulta e descarregamento dos títulos inseridos nas Bibliotecas Digitais UC Digitalis, UC Pombalina e UC Impactum, pressupõem a aceitação plena e sem reservas dos Termos e Condições de Uso destas Bibliotecas Digitais, disponíveis em https://digitalis.uc.pt/pt-pt/termos.

Conforme exposto nos referidos Termos e Condições de Uso, o descarregamento de títulos de acesso restrito requer uma licença válida de autorização devendo o utilizador aceder ao(s) documento(s) a partir de um endereço de IP da instituição detentora da supramencionada licença.

Ao utilizador é apenas permitido o descarregamento para uso pessoal, pelo que o emprego do(s) título(s) descarregado(s) para outro fim, designadamente comercial, carece de autorização do respetivo autor ou editor da obra.

Na medida em que todas as obras da UC Digitalis se encontram protegidas pelo Código do Direito de Autor e Direitos Conexos e demais legislação aplicável, toda a cópia, parcial ou total, deste documento, nos casos em que é legalmente admitida, deverá conter ou fazer-se acompanhar por este aviso.

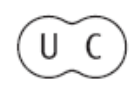


CONIMBRIGA

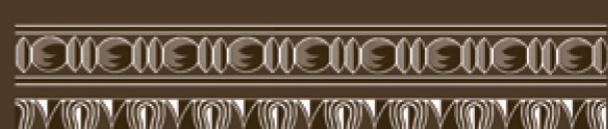

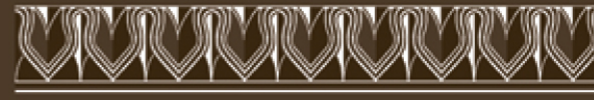

INSTITUTO DE ARQUEOLOGIA

VOLUME LIII • 2014

FACULDADE DE LETRAS 


\begin{abstract}
Alessia Amato
Comune di Ruvo di Puglia - Tavolo Tecnico del PUG, Carta Arqueológica e Valorização do Património. Collaboratore Esterno per la Soprintendenza - Archeologia della Puglia

alessiaamato@inwind.it
\end{abstract}

\author{
ARCHEOLOGIA DELLA NAVIGAZIONE ISLAMICA \\ D'OCCIDENTE: LO STUDIO DI CASO DEL GHARB PORTOGHESE
}

\author{
ARCHAEOLOGY OF THE WESTERN ISLAMIC NAVIGATION: \\ THE STUDY OF THE PORTUGUESE GHARB CASE \\ “Conimbriga" LIII (2014) p. 201-223 \\ http://dx.doi.org/10.14195/1647-8657 53_ 7
}

ABSTRACT: The compilation of the documentation previously referred embraces several fields of the definite research of the Meio Acquático (Water World), topography and geomorphology, literature and history and not for last, archeology. In the archeology field we will particularly enunciate all the leading to draw the final environment of the watercraft (boat); presumably the Islamic Qarib, the contact vehicle in several cultural and technological moments.

KEYwORDS: water world; Islamic; watercraft

RESUmo: A compilação global do trabalho define o limite da pesquisa dentro da acepção de Meio Aquático, tanto no sentido topográfico quanto geomorfológico, junto com os aspectos literários e históricos e mais propriamente arqueológicos. É na arqueologia que se foca o presente contributo, em particular na funcionalidade das embarcações, ou seja do presumível Qarib Islâmico, veículo do contacto cultural, técnico quanto metodológico, na esfera da construção naval.

PALAVRAS-CHAVE: meio aquático; período islâmico; embarcações

Conimbriga, 53 (2014) 201-223 
RIASSUNTO: La linea conduttrice del lavoro si impone tra gli aspetti topografici e geomorfologici, oltre alle caratteristiche Letterarie e Storiche nonché propriamente archeologiche. É proprio nell'archeologia che si focalizza il presente contributo, in particolar modo nella funzione delle imbarcazioni, ovvero il presumibile Qarib islamico, veicolo del contatto culturale, tecnico quanto metodologico, nella sfera della costruzione navale.

PAROLE CHIAVE: mezzo acquatico; periodo islamico; imbarcazioni 


\section{ARCHEOLOGIA DELLA NAVIGAZIONE ISLAMICA D'OCCIDENTE: LO STUDIO DI CASO DEL GHARB PORTOGHESE}

\section{Introduzione alle componenti della disciplina}

Lo studio da cui proviene questo contributo ${ }^{1}$ determina le sue caratteristiche entro la sfera cronologica della presenza mussulmana della Penisola Iberica e nello specifico del Garb sud portoghese, più propriamente nel territorio compreso tra basso Tejo ed Algarve. L'analisi della relazione di questo con il mare e tutte le sue componenti, precede il periodo dell'Espansione cinquecentesca, momento in cui il cristiano Regno di Portogallo affronta l'Oceano, ricontestualizzando il bacino del Mediterraneo ed i circuiti classici che avevano detenuto la totale attenzione della navigazione sino a quel momento.

$\mathrm{Nel}$ periodo islamico preso in considerazione, tra i secoli VIII e XIII, la vitalità dei centri portuari e lo sviluppo delle attività chiaramente nautiche, quindi la cantieristica navale, sono spesso richiamate dagli scritti degli autori arabi, tra cui i noti Al-Razi, Al-Bakri o Idrisi per citarne alcuni. Assumendo che i testi letterari, quindi, costituiscano il corpus della geografia medievale utile allo studio della costa e delle rotte di navigazione, le città marittime restituiscono una grande quantità di rinvenimenti, sottolinenando la persistenza delle rotte commerciali e l'utilizzo regolare degli approdi marittimi e fluviali.

È altresì importante ricordare che le testimonianze del mondo marittimo islamico sud peninsulare, si sviluppano a partire dall'analisi interdisciplinare delle sue svariate componenti. Il loro utilizzo compensa la mancata conservazione di elementi archeologici e l'articolato

${ }^{1}$ Lo studio qui presentato è parte dei risultati della Tesi di Dottorato, ricerca condotta presso l'Università di Coimbra, Facoltà di Lettere, con borsa individuale finanziata dalla Fundação da Ciência e Tecnologia.

Conimbriga, 53 (2014) 201-223 
processo di confronto con i tratti etnografici attuali, manifestando la continuità delle pratiche ancestrali ed utilizzo ininterrotto degli stessi luoghi. In questo senso interviene, per esempio, la cartografia antica comunemente utilizzata per lo studio dei cambiamenti costieri, presupposto fondamentale per il riconoscimento delle antiche installazioni, obbligando così a una riflessione sul concetto stesso di spazio portuario. Gli attracchi e i luoghi in relazione con gli scambi commerciali e culturali, nel mondo post-lusitano, sarebbero i luoghi per eccellenza del contatto con l'informazione tecnica navale, indicando la strada per la loro diffusione e materializzazione negli spazi destinati alla costruzione navale, attracchi piuttosto semplici, cantieri o arsenali.

Nel parlare di periodo medievale islamico, con particolare riferimento ai ritrovamenti marittimi, l'interpretazione di questi si distanzia dalle letture oltrepassate secondo cui, in una visione baratiana quanto meno, le limitazioni dell'archeologo navale risiederebbero nella mancanza dell'oggetto di studio, quindi l'imbarcazione. Con la stessa parzialità interpretativa, si era a lungo guardato alla navigazione del periodo romano. Piuttosto si sarebbe dovuto riformulare il canone identificativo che ha sofferto dell'imprecisione con cui i natanti, in mancanza di riscontro, venivano definiti genericamente medievali, pregiudicando quindi la corretta interpretazione dell'apparecchio arabo-islamico.

La lingua araba, però, si attesta nella tecnica e nella comune parlata marinaresca. Questa era la formula comunicativa più diffusa tra i commercianti, il mezzo praticamente attuato per il transito terminologico e tecnologico, la lingua del contatto tra gli antichi occupanti del sudest della penisola ed i nuovi conquistatori, dinastie rinnovate del territorio portoghese. Si pretende solo un accenno, in questo scritto, all'ampia sezione dedicata nel corso degli studi alle interpretazioni iconografiche provenienti dalle forme ceramiche più note, ovvero bacini ceramici e forme aperte comprese tra l'area pisana e quella maiorchina. Queste sono dunque utili all'identificazione di talune caratteristiche del tipo di imbarcazione in esame, dovutamente confrontati con i più approfonditi naufragi coevi del mediterraneo, in particolare occidentale.

\section{Il contesto storico e metodologico}

Le imbarcazioni islamiche devono aver dominato il Mediterraneo con un picco accentuato tra i secoli XI e XIII. In questa visione

Conimbriga, 53 (2014) 201-223 
di traffici e scambi, ricalcante le rotte pregresse, non sarebbe corretto immaginare tripulazioni dotate di punti esatti riportati sulle carte o corrispondenze dettagliate degli astri. I tratti grafici delle mappe avranno infatti avuto un utilizzo parziale, quasi un promemoria dei punti e delle distanze tra gli stessi, in particolar modo nell'orientamento rispetto alla linea di costa e all'approdo nei relativi scali. Allo stesso modo non sono recuperabili dettagli certi sui diversi tipi di tecniche costruttive, così per ottenerne utilizziamo le componenti iconografiche e delle rappresentazioni temporali, in un utile confronto con le testimonianze artistiche seguenti. Come già detto, nel focalizzarci sul periodo islamico, si considera come riferimento imprescindibile lo spoglio ceramico del Mediterraneo Occidentale, elementare dimostrazione del contatto con la sfera acquatica orientale, nonché con l'altalenante mondo bizantino.

Per meglio contestualizzare il registro iconografico, si prendono qui in considerazione le forme ceramiche aperte, tra bacini pisani e ataifores maiorchini, e il loro confronto con i materiali di archivio, in riferimento alle navi ad un solo maestro e velame latino o alla trina con sistemi di governo armati in maniera assiale ${ }^{2}$.

Il qārib preso in esame, appare quindi conseguenza dell'accostamento di diverse necessità impossibili da racchiudere in un'imbarcazione univoca, tenendo in conto la molteplicità delle formule attuabili nella fase di costruzione e messa in opera di un'imbarcazione. Spesso queste dipendono esclusivamente dalla necessità interpretativa del realizzatore e dal suo bagaglio di esperienze. La concezione quindi secondo cui lo studio seppur esaustivo dei relitti noti, possa condurre ad un tipo unico di natante, deve confrontarsi con la molteplicità dei relitti ancora in fase di analisi o peggio non più fruibili. Come detto, la metodologia impone una cautela proveniente dalle variabili nella componentistica, spesso personale, della mano realizzatrice, del luogo e del materiale di produzione o ancora dell'arsenale di provenienza. Le fonti cartografiche inducono ad una riflessione con base postuma rispetto alla cronologia islamica, comparando le Vedute Panoramiche, molto in voga sin dal $\mathrm{XV}$ secolo, ed il loro coronamento di parchi nautici, il cui confronto in negativo consente di evidenziare la mancanza della tipologia indaga-

\footnotetext{
${ }^{2}$ Non si esporrà in questa sede, nonostante l'ampia elaborazione nel corso degli studi, sulle componenti relative alle raffigurazioni graffite provenienti dal sito fluviale di Mértola, nel sud portoghese. Si rimanda in bibliografia a più specifici riferimenti.
}

Conimbriga, 53 (2014) 201-223 
ta. In secondo luogo, l'iconografia coeva, documentazione coroplasta e attestazioni popolari di arte estemporanea, come i citati graffiti, oltre alla continuità di elementi puramente cantieristici nella costruzione tradizionale lignea, sono stati identificati quali perni evolutivi che potessero condurre, senza pretese di riuscita, alla più nota ed acclamata Caravella portoghese. I quesiti che ne derivano si legano alle contaminazioni nell'evoluzione tecnica, lì dove una metodologia mediterranea possa avere intersezioni con i contatti nordici tramite atlantico (karvel o caravel), quindi consentire un radicamento tipologico della suddetta imbarcazione, ascrivibile al sud del Portogallo, già Garb. Di qui tutte le riflessioni sull'avvento della vela latina nell'importante sistema costituito dalla rotta atlantica, attestata sin dal periodo romano su tratti di civiltà precorritrici la stessa, evoluzioni di affiancamenti orientali nel già documentato XVI secolo, esame delle metodologie direttrici, dai governagli latini ai timoni assiali, e quei tratti ancora percettibili nelle Faluas algarvie del XX secolo.

\section{Indici di contaminazione linguistica nella tecnica navale}

Le navi mussulmane ambirono al dominio del Mediterraneo, con un notevole slancio tra IX e X secolo, unendo le sponde dell'Andalus, quindi il Garb e Magreb (Picard 1997), slanciandosi sotto altre spoglie sino in Cina ed ai porti in contatto con le opulenti realtà di Aden e Jeddah. Nel Mediterraneo però, le imbarcazioni da guerra, perlomeno sino al primo periodo dell' Islam, mantengono ancora una struttura in cui lo sforzo propulsivo è concentrato verso gli elementi portanti, sulla linea delle ordinate mastre. Ne consegue una maggiore lentezza, dinanzi alla quale lo stimolo alla velocità è recepito dalle migliorie costruttive tra VI - XII secolo. Le miniature delle Chronicle di Joannes Skylitzes, fr. c.1081, mostrano una forte similitudine tra navi islamiche e bizantine, tanto da dover ricorrere alle indicazioni grafiche sovrastanti per differenziarle ${ }^{3}$. Sulla stessa scia di parzialità documentale, sarebbe inutile riaccendere la già ampia contesa sulla supremazia o efficacia di metodi di assemblaggio a mortase e tenoni o cuciture. Nel contempo, le dinami-

\footnotetext{
${ }^{3}$ Sito presso la Biblioteca Nacional de Madrid, in Tselikas 2000 apud Agius 2008.
}

Conimbriga, 53 (2014) 201-223 
che di diffusione culturale consentono ai popoli in contatto di adottare idee astronomiche e tecniche, che come riferisce McPherson fomentano l'ambizione a risultati di ispirazioni indipendenti (MCPHERSON 1995: 33). Il timone assiale per esempio e la sua irruzione nel Mediterraneo, XI-XII secolo all'incirca, avviene per mezzo del solito Mar Rosso, o la vela triangolare, adottata dai bizantini che avrebbero ritenuto questa una valida soluzione alla navigazione di bolina, nonostante la precisa provenienza con gli apporti tecnologici risulti alquanto speculativa. ${ }^{4}$

L'aspetto più propriamente terminologico su cui si vuole incentrare l'attenzione è quello compreso tra i tre termini Safina, qārib, markab. Il primo termine, noto nelle cronache e negli scritti letterari, é spesso ricollegato al dromedario ed al cammello, nella loro definizione di Nave del Deserto, Safina al-barr, metafora dal mondo letterario (AgIUs 2008: 270). I Qārib/qawārib, intorno al IX secolo, sono considerate imbarcazioni di servizio, forse sulle indicazioni di Ibn Battuta che con $q \bar{a} r i b$ definiva un insieme di piccoli natanti di appoggio, capienti. La radice araba $q \cdot r \cdot b$, proverrebbe infatti dal verbo avvicinarsi ${ }^{5}$. I termini andalusi $q \bar{a} r i b$ o qărab, precedendo le portoghesi e spagnole caraba e carabo, XIII secolo, dal latino carabus a sua volta diffusosi in Egitto, Siria e Mesopotamia (Pellegrini 1978 II: 817-818) da quella derivazione greco-bizantina Karabós. Gli usi però sembrano comprendere un insieme di imbarcazioni generiche, legate al mondo islamico-bizantino, spesso in accezioni belliche. Tra i tanti toponimi cui potremmo far riferimento, evoluzioni da un capo all'altro del mondo apparentemente sconnesse, è il caso di nominare il tipo 'Ushärī, una sorta di gondola con copertura a botte. Una raffigurazione attinente a questo tipo di imbarcazione potrebbe essere quella del grande vascello inciso nei graffiti di Alcácer do Sal, potrebbe corrispondere a questa tipologia fluviale, nell'estremo oriente solitamente di connotazione regale (AL-BAGHDADI, nd. 54, apud Agrus 2008: 301). Una nave da guerra era invece lo shalandi (Dozy 1967 I: 783), una tra le tipologie bizantine, di provenienza greca, kelàndion o kelàndra, poi chaland francese e scialando italiano, mantenendo una singolare coincidenza con il termine dialettale siciliano, ossia lasciarsi trasportare lentamente dagli avvenimenti. E

${ }^{4}$ È direzione interpretativa proveniente dai fervidi ambienti dell'Indico. Biblio Roxani, M 2002 o Blot JY, 2003.

${ }^{5}$ In questa come in altre sedi i ringraziamenti più sentiti alla Dottoressa Augusta Nunzia Mastropasqua per l'ausilio nella percezione terminologica araba.

Conimbriga, 53 (2014) 201-223 
ultimo il calão portoghese, larga e lunga imbarcazione con circa dieci, dodici remi per fiancata, utilizzato nella pesca del tonno nonché definizione di atteggiamento lento e trascinato ${ }^{6}$.

Lancia-fiamme, o chiatte con materiale infiammabile e metodi propulsivi ad aria per espandere lo stesso, sono testimoniati da Ibn al-Athir nel XIII secolo, con riferimento alla campagna di Siracusa, attribuendo la derivazione fonetica al verbo $h \cdot r \cdot q$, distruggere (PICO 1963), divergendo in parte con la funzionalità della carraca, eventuale evoluzione lessicale. Ancora una nota prima di lasciare spazio al confronto iconografico ed ai termini imposti dai ritrovamenti subacquei. In Portogallo l'evoluzione del termine caravella é stato per tempo affrontato, riconoscendovi diverse inflessioni. Qārib avrebbe costituito la radice della diramazione carabo e caravela, piuttosto che caravela diminutivo di carabo (NASCENTES 1932, apud COROMINAS 1954-57 I: 662). La trasformazione semantica francese, come Alcorel accenna nella su Cronica de Jerusalem nel 1930, ne designa una imbarcazione piccola (PICo 1963: 74). Mancano indicazioni utili sulla stazza nell'arabo-ispanico, secoli XI - XIII, magrebino medievale e catalano antico, XIV secolo, tutte inclini nel riconoscere al termine caréu, il significato d'imbarcazione generica da trasporto ${ }^{7}$. L'accezione femminile sarebbe poi derivata dalla diffusione avvenuta con l'opera Partidas, Alfonso X -1256-1265, a cui il suffisso - ela, ne avrebbe aggiunto il diminutivo, o come ne affermava Lopes de Mendonça, la fusione, caravo-à-vela, per designarne la propulsione.

Senza addentrarsi ulteriormente in questo esercizio comparativo, sulla soglia delle accezioni regionali, la visione di Oliveira Marques sembra offrire quella chiave di lettura, condivisa, che consenta di ragionare sulle evoluzioni semantiche -No Algarve de 1320-21 continuavam a predominar os mouros e as mourarias e as mesquitas avantajavam os templos cristãos, era uma zona de fronteira em processo de cristianização, uma autêntica colónia (FonSECA 2003: 59). Una colonia appunto, linguistica e tecnica.

\footnotetext{
${ }^{6}$ Suggerimento terminologico, gentilezza del Prof. Dottor Vasco Mantas, Universidade de Coimbra.

${ }^{7} \mathrm{E}$ in questo periodo che per la prima volta in un documento portoghese viene nominata una caravela, con accezione femminile; Alfonso III nell'opera Partidas (12561263; in Pico 1963: 74).
}

Conimbriga, 53 (2014) 201-223 


\section{La nautica specchiata: iconografia delle forme ceramiche}

Il periodo medievale islamico fa riferimento alla rappresentazione con un'enfasi nettamente superiore alla nostra percezione. La diffusione di testi dal carattere sociale impongono al tratto scritto la decorazione grafica, spesso occupando intere parti di pagine e fogli. Allo stesso modo sembra che la decorazione a tutto tondo delle forme ceramiche aperte, bacini o ataifores, abbia avuto lo stesso intento dell'apertura a tutta pagina delle miniature. Gli avvenimenti giornalieri non avrebbero avuto motivo di essere considerati e fermati nelle illustrazioni, contrariamente ad annotazioni da sottolineare caratterizzando il committente o un particolare momento. Se per esempio nella restituzione di un trasbordo fluviale di grano, direttamente dalla stiva in sacchi di tela sollevati a spalla, si scorgesse una tipologia nautica, la raffigurazione ne sarebbe motivata dall'esaltazione delle caratteristiche commerciali di un dato signore, non del trasbordo in sé. É per questo che la voracità con cui captiamo gli elementi dei natanti potrebbe divergere dalla loro reale struttura.

Con il fine di determinarne gli elementi si presenta in questa sede, il confronto tra i Bacini $n^{\circ} 19, n^{\circ} 59, n^{\circ} 292$ provenienti dal Museo Nazionale di San Matteo, $\mathrm{Pisa}^{8}$, l'ataifor di Dénia, Museo Arqueológico de Dénia, M.I. Ayuntamiento de Dénia ${ }^{9}$ l'Ataifor ceramico proveniente dal museo ARQUA di Cartagena, appartenenti alla sfera cronologica compresa tra la fine del X e XI secolo, con forti caratterizzazioni maiorchine. Temporalmente posteriore, la ceramica simile arabeggiante con raffigurazione di imbarcazione portoghese, è attinente alla caratterizzazione come unione tra le tradizioni, perlomeno a livello grafico: Malaga 1425-1450, ceramiche invetriate esportate sino all'Egitto o alle coste inglesi, probabile glorificazione dei successi di un commerciante, attualmente presso il VAM di Londra, $\mathrm{n}^{\circ} 486-1864$. Questi in connessione visiva con tutti gli elementi traibili dagli studi sui rinvenimenti e naufragi noti.

Un'ultima specifica prima di affrontare gli elementi tecnici a nos-

\footnotetext{
${ }^{8}$ Presso le strutture museali pisane, si è avuto modo di conoscere personalmente e di giovare degli insegnamenti della Dottoressa Berti Graziella, direttrice del Museo e profonda conoscitrice delle dinamiche commerciali del Mediterraneo Occidentale. Alla Sua memoria sono dedicati infiniti ringraziamenti.

${ }^{9}$ Dati gentilmente concessi dal Dottor Felipe Castro.
}

Conimbriga, 53 (2014) 201-223 
tra disposizione. Le restituzioni grafiche risentono, come É ovvio delle deformazioni a cui l'artista è costretto, in ampliamenti e dilatazioni spaziali o perpetrare di tipologie non contemporanee. Non sembrerebbe essere questo il caso degli elementi presi in considerazione. Inoltre è importante sottolineare che non tutti gli elementi più peculiari per definire una tipologia nautica, possano essere sondati in raffigurazioni di cui l'opera morta è principale veicolo di informazioni.

Tra gli elementi tecnici più importanti, la chiglia, che indica quel tratto continuo esteso tra poppa e prua, collegamento con le ossature trasversali dello scafo, la cui lunghezza varia secondo le misure complessive dell'imbarcazione. Una nave, di qualsiasi tipologia, non ne può prescindere, eccezion fatta per alcune innovazioni dell'era contemporanea. Si potrebbe ricercare un tratto piano di questo elemento nel Bacino $\mathrm{n}^{\mathrm{o}} 59$, forse indicativo dell'utilizzo di un unico legno sormontato dalle ruote di prua e poppa. Questi elementi corrispondono ad ognuna delle sezioni di una nave. La loro lunghezza varia a seconda della posizione occupata, diminuendo dall'ordinata maestra in direzione di poppa e prua. Lo spazio dello scheletro compreso tra la maestra e la poppa, dove si colloca il timone, è definita rè dal termine latino rètro in uso nel Mediterraneo Occidentale e di forte influenza francese; l'area delimitata dalla prua invece è definita avante, anche questa da un'accezione francese del XIV secolo (PICO 1963: 221-222). La pompa di sentina, impossibile da scorgere, viene invece citata per la prima volta in un documento Foral 18 Agosto1410 definita arca de bomba ${ }^{10}$. La sua funzione è quella di aspirare l'acqua che entra nella stiva dove è collocata, solitamente al di sopra della chiglia, bloccata dalle serrette. Il documento dell'inizio del XV secolo fa presupporre il suo utilizzo anche in periodi precedenti. Il termine arca veniva spesso sostituito nelle diverse accezioni nautiche iberiche con il vocabolo cesto de gàvea (MorAIS 1948-1959 apud Pico 1963: 247), determinazione lentamente abbandonata appunto a favore della definizione di arca, elemento questo appartenete all'armamento della chiglia e non al sistema di alberi. Questi elementi sono infatti, tutti limitrofi lo scheletro, chiglia o paramezzale, elemento appena sovrastante. Nei bacini $\mathrm{n}^{\circ} 19$ e $\mathrm{n}^{\circ} 59$, la prua è fortemente arrotondata con una siluette a falce di luna campita, completata da un reticolo fitto. Già in queste estremità è possibile notare qualche

${ }^{10}$ Marques 1944 Foral 18 Agosto 1410, I vol.

Conimbriga, 53 (2014) 201-223 
elemento contiguo con l'imbarcazione del piatto di Malaga, con uno strascico nelle imbarcazioni del Nord del Portogallo, potendone quindi attribuire una ruota molto alta. Altrettanto importante è la definizione del timone, apparecchio sito a poppa dell'imbarcazione fondamentale per la direzione. Altre due parole impiegate nel medioevo per designarlo erano governale e peltre, termini che sembra siano coesistiti perlomeno fino al XV secolo I documenti relativi a questo periodo li citano indistintamente ${ }^{11}$. Il governale è il timone a pala laterale usato già nella marineria antica, probabilmente da età protostorica, singolo o doppio, governall nel catalano antico, dal latino tardo gubernaculu. È citato in documenti dal XIV secolo in poi, il che rende implicito un suo utilizzo in periodi anteriori. Un documento Foral 1440 Março 8, caso vi fossero incertezze, ci informa che il luogo di questo era na popa (MARQUES 1944 vol. I ; PICo $1963: 315$ ). A supporto di questo utilizzo interviene il bacino pisano $\mathrm{n}^{\circ} 292$, e un probabile tratto nel bacino $\mathrm{n}^{\circ} 19$, in fuoriuscita dal castello di poppa. Nel confrontare i negativi dei pezzi ceramici $\mathrm{n}^{\circ} 19$ e $\mathrm{n}^{\circ}$ 59, elementi decorativi della Chiesa di San Pietro a Grado, si nota che l'imbarcazione potrebbe manifestare la presenza di un timone assiale incluso dall'esecuzione grafica della poppa. Questo elemento non è visibile nell'ataifor di Dénia poiché fortemente lesionato. Una contemporaneità dei due sistemi di conduzione del qarib, a seguito poi raffrontati con la sagoma disegnata dal Maestro João de Lião (BARATA 1996 vol. I: 20 FIG. 2), senza però forzare la lettura di una continuità evolutiva che porrebbe l'attenzione sulla nave di Malaga, nella ceramica del XV secolo.

Per la copertura dello scafo, si sfrutta l'assemblaggio di un certo numero di travi lignee che costituiscono i bordi dell'imbarcazione e ne delimitano la forma nelle metodologie a scheletro portante: è questo il fasciame. I due termini più comuni in apporto sono bordalha e madeira. Singolare notare come lo stesso si tramuti nell'accezione negativa di gente abbietta (PICO 1963: 183), contrariamente al madeira nell'area occidentale del Mediterraneo e prima fascia atlantica portoghese. In una sovrapposizione a raggi $\mathrm{X}$ del registro inferiore del corso dello scafo, è possibile notare linee oblique su curvature parallele, da cui si ipotizzerebbe un corso del fasciame embricato, a clinker, più visibile nel bacino $\mathrm{n}^{\circ} 19$ che nel $\mathrm{n}^{\circ} 59$. Anche questo dato è illeggibile nella

${ }^{11}$ I documenti relativi a questo periodo li citano indistintamente.

Conimbriga, 53 (2014) 201-223 
breve restituzione di Dénia. Continuando nella ricerca degli elementi strutturali, il capo di banda sembra delimitato dall'ispessimento lineare più chiaro, forse una cinta di rinforzo a prua o sistema a sostegno del castello, nei bacini $\mathrm{n}^{\circ} 19, \mathrm{n}^{\mathrm{o}} 59$, nel tratto di Dénia. Fortemente marcante nella visione a raggi $X$ dello scafo del bacino pisano $\mathrm{n}^{\circ} 292$, se si vuole quello più cristiano del congiunto. L'inflessione delle ruote apporterebbe più elementi per un confronto con una cocca che con un qarib. Il velame è altresì riscontrabile solo per mezzo di restituzioni grafiche, cui si aggiungano gli alberi di sostegno delle vele, il cordame e la fattura delle vele stesse, mantenendo simili le modalità di ammainamento. Si scorgono antenne trasversali all'albero, in pennoni agganciati in maniera obliqua, fissati al maestro elevandosi a circa due terzi dell'altezza complessiva. Il termine maestro è chiaramente riferito all'albero centrale il cui piede poggia sulla scassa. Più vele nei bacini $\mathrm{n}^{\circ}$ 19 e $\mathrm{n}^{\circ} 59$ e ataifor di Denia ed in quello di Cartagena che ne accenna addirittura tre, tutte queste alla trina. Una portante nel piatto di Malaga, quadra, una evidentemente ridotta nel bacino $\mathrm{n}^{\circ} 292$, ad un solo albero, forse la derivazione di un artimão greco. Cavi e cime legano le vele ai bordi dello scafo. Si tratta probabilmente di nodi bastardi, che prendono le verghe ai maestri, e boline, cavi che fissano le vele ai bordi, ed una scocca nel bacino $\mathrm{n}^{\circ} 59$. Ultimo elemento dell'alzato della nave, non meno importante, é il castello di poppa, anche detto per le evidente attinenze difensive, castrum - al-qaçbâ, presente a grandi linee nei pezzi ceramici pisani $\mathrm{n}^{\circ} 19$ e $\mathrm{n}^{\circ} 59$, non presente per Dénia né Cartagena viste di prua, forse riconoscibile nel piatto di Malaga, appena sovrastante il reticolato di contenimento, una sorta di balaustra, lì dove sono impiantati gli stendardi triangolari. Un ultimo elemento da segnalare é la tettoia o copertura per le merci, presente nei bacini $\mathrm{n}^{\circ} 19$ e n ${ }^{\circ} 59$, nell'ataifor di Dénia, appena accennato in quello di Cartagena. L'assenza negli altri due elementi può essere spiegata nella seguente forma: il bacino $\mathrm{n}^{\mathrm{o}} 292$, potrebbe non aver avuto bisogno di coperture forse a causa della sua peculiarità peschiera, o per la probabile necessità di rappresentare la struttura interna dello scafo, qualora fosse usato per il carico di merci con questo curioso sistema di raffigurazione in trasparenza ${ }^{12}$.

${ }^{12}$ Il Dottor Paul Arthur, Università degli Studi del Salento, altresì ne identifica una lettura a Raggi $\mathrm{X}$ in cui intravedere il colonnato di una navata, quindi un suggerimento religioso.

Conimbriga, 53 (2014) 201-223 
Lo scafo di Malaga altresì sembra caratterizzato da più coperte, quindi spazi appropriati di stivaggio.

Tenendo in conto la diffidenza che questo tipo di lettura soffre, quella del mancato raffronto diretto, è però possibile attribuire una certa continuità perlomeno stilistica nel riempimento della forma rotonda dei piatti, con immagini ittiche, tonni probabilmente, e scritte glorificatrici e propiziatorie. Nulla che manchi al resto delle culture marittime a livello globale, non volendo tralasciare la seppur remota possibilità di attestare, esclusivamente con un ritrovamento archeologico adeguato, la possibilità che i pesci rappresentati non fossero in realtà che una parte fondamentale del carico, quindi che le stesse imbarcazioni fungessero da trasporto di pescato di grande stazza poi rivenduto in altra sede portuaria, insieme a merci deperibili della produzione locale $^{13}$, La preservazione di un qualsiasi scafo naufragato in pieno Atlantico, privo di carico soggetto a concrezioni, risulta come già argomentato altamente complesso.

\section{Coordinate dell'Islam naufragato}

L'intento di identificare una certa tipologia di imbarcazione, avviene in primo luogo su base etimologica tenendo però conto del giusto grado di diffidenza che ci lega alla sua diffusione giacché la contaminazione e continuità risentono delle accezioni dialettali, facendo maggiore affidamento su evidenze documentali e riscontro archeologico. In primo luogo le necessità dell'evoluzione del trasporto avranno forzatamente condotto ad una modifica del tonnellaggio, non più relativo al carico anforario ma sì alla capacità della stiva, consequenziale al progressivo sempre maggiore utilizzo dei tonees, le botti. Qui si imporrebbe una riflessione sul tipo di carico, suggerita dalla raffigurazione dei Bacini presi in esame, nonché di un ricorrente linguaggio ittico su altre forme ceramiche. Il pescato, i tonni di grandi dimensioni, potrebbero essere parte integrante di un'imbarcazione con carico eterogeneo, specialmente in viaggi per concezione lunghi e strutturati in varie soste. Non sarebbe nuovo questo modello di diffusione, già la Lusitania ne

\footnotetext{
${ }^{13}$ La possibilità si basa sulla tradizione ittica ed il trasporto in epoche pregresse di pescato non lavorato, soggetto quanto più ad una prima salagione in stiva.
}

Conimbriga, 53 (2014) 201-223 
aveva fatta un'arte con la divulgazione della salagione e della sardina ${ }^{14}$. L'espansione linguistica e tecnica quindi, devono essersi intersecate nonostante la profonda difformità geografica che porta a considerare con cautela una linea unica di contatto. Lo scambio avveniva su tratte già percorse dai geografi, spostando merci, pellegrini, e stimoli tecnici in importanti corridoi da e verso l'Oriente Estremo, attraversando il Mar Rosso e accrescendo i traffici con il Mediterraneo. Le classificazioni di imbarcazioni, alla luce di questo contatto, sembrano gradatamente allontanarsi dalle macro tipologie, anche stereotipate, secondo cui ai bizantini corrispondesse il Dromon ${ }^{15}$, ai vichinghi il Drakar ed ai restanti popoli del Mediterraneo, fatta eccezione per i legni da guerra, una sorta di karabós di evoluzione latina, capiente e lento. Tipologia e metodologia nella messa in opera, così come la tecnica di assemblaggio, sono diffuse in maniera capillare, presentando una mescolanza confermata dai relitti, nonostante la non sempre possibile lettura dei dati subacquei. Le fonti documentali, per quanto adattate alle contingenze, sono ottimi spunti di riflessione e confronto, testimonianza di una cultura del mare innegabile.

Spostando l'esercizio ricostruttivo su base comparativa, si potrebbe dedurre la formula di un qārib islamico da commercio, intorno ai 100 Tonn, con vele alla trina e governo gestito da un timone assiale movibile, con scafo calafatato e seguenti caratteristiche, dalla lunghezza, all'incirca di $20 \mathrm{~m}$. La media é ottenuta dai relitti di Agay, Le Bataiguer scafo $\mathrm{n}^{\circ}$ 1, Camarina C, Culip F, Marsala A, Pélagos, Serçe Limani A (PARKER 1992; BASS STEFFY et alii 2002). Questo scafo, più che studiato, presenta caratteristiche interessanti tanto sul piano strutturale che del contenuto. La complessa origine di carico ed equipaggio fornisce una buona visione d'insieme. Lo scafo è rotondo a fondo piatto, alte le fiancate, rinforzate all'interno e a scafo portante, con tavolame assemblato a paro. In relazione agli altri naufragi però, la messa in opera prevalente è a scheletro portante, come nel Culip $\mathrm{F}$, associata a una tecnica a mortase e tenoni, rinforzati da chiodi in ferro, come nei casi di Agay (JÉzÉGOU 1997), Arenella, Megadium B, Plane C. Il massiccio di

${ }^{14}$ Con riferimento agli studi dottorali in corso di Bombico Rúpio S.A., Universidade de Évora.

${ }^{15}$ La riflessione è obbligatoria: il termine greco Dromon, dal termine dromedario, quindi corridoi, si allineerebbe etimologicamente all'evoluzione linguistica di Dhoni, imbarcazione tipica delle Maldive, ed. Alves 1998: 71.

Conimbriga, 53 (2014) 201-223 
scassa è evidente nei relitti di Marsala A e Nin A e B, nonostante questi ultimi possano piuttosto essere affiancati ad una tipologia snella, quella probabilmente espressa nei graffiti di Mértola A e B, avendo in dotazione, nella parte interna dello scafo, delle costole utili sia a srotolare più facilmente le sartie sia a bloccarne i remi, tutti indizi di una navigazione rapida, d'attacco. Infine, rileviamo l'interessante possibilità di riscontrare la dinamica della navigazione in convogli, suggerita da Picard, in corrispondenza dei relitti di Agay, Le Bataiguer (JonCHERAY 1975, pp.42-48), Marsala A B e C, Nin A e B.

La possibilità quindi, di attribuire la modalità rappresentata dai bacini $\mathrm{n}^{\mathrm{o}} 19$ e $\mathrm{n}^{\circ} 59$, ossia quella di affiancare alla navigazione imbarcazioni di minori dimensioni, è da prendere in considerazione per il sito del naufragio di Marsala (A e B), prototipo a grandi linee di ciò che si pensa possa essere stato un $q \bar{a} r i b$ islamico tanto nel Mediterraneo, quanto nel Garb al-Andalus.

In conclusione, nonostante l'assenza in questa formulazione del riferimento ai luoghi di approdo e produzione navali tra prima fascia Atlantica e Mediterraneo, si ha modo di inserire il Garb portoghese nel sistema affannato del Mare Nostrum, cerniera dall'Occidente all'Oriente delle peregrinazioni. Il contatto tra questi lembi specchiati, concretizza in esperienza l'utilizzo di sommarie carte e portolani. In questi mari gli scafi fagocitati dalle acque, con o senza carico, precedono l'avvento delle Repubbliche Marinare. I natanti forniscono uno scenario degno al tentativo di ricostruire quella tipologia di imbarcazione da commercio, agile e capiente, rispondente alle direttive della navigazione mediterranea. La stessa imbarcazione mostrerà una propensione al cambiamento strutturale, in rotte altre, sotto spinte longitudinali provocate da mari atlantici e navigazioni stagionali. Sarebbe questo il caso del qārib, connotato solidamente sotto il profilo linguistico e successiva diffusione dialettale, mutevole in termini di utilizzo, risultato di una formulazione del contatto tecnologico. La componentistica dei relitti e le peculiarità di una costruzione formulata sull'esperienza individuale, apre la strada ad una restituzione nautica su base ceramica. Tutti questi elementi palesano quindi, senza pretesa di riuscita, l'intento di segnare il passo della tradizione lignea, fissando nel periodo islamico di interesse, il momento di passaggio cantieristico che conduca alla più acclamata Caravella portoghese. 


\section{BIBLIOGRAFIA}

Agrus, Dionisius A. (2008) - HdO Classic Ships of Islam, from Mesopotamia to the Indian Ocean, Brill ed. Leiden, Boston.

Alves, F. J. (ed.) (2001) - International Symposium on Archaeology of Medieval and Modern Ships of Iberian-Atlantic Tradition. Trabalhos de Arqueologia 19, Lisboa, IPA.

Barata, J. P. G. (1996) - Estudos de Arqueologia Naval, 2 vols., Imprensa Nacional, Casa da Moeda, Lisboa.

Bass, G. F. S. e Mattews, S. (2004) - Limani: An Eleventh-centuary Shipwreck, Texas A\&M University Press.Volume 2.

BLot, Y. (2003) - The Imperial Bamboo, Cultural Institute of the Macao SAR Government.

Corominas, J. (1854-57) - Dicionario Critico Etimilogico de la Lengua Castellana, 4 vol., Madrid Ed., Madrid.

Dozy, Reinhart, P. A. (1967) - Supplément aux dictionaires arabes, vol. I-II, Leiden, Brill (ried. 1887).

Fonseca, P. Q. (2003) - As origens da Caravela Portuguesa, Chaves Ferreira Publicações, S. A., Porto.

JÉZÉGOU, M. P. et alli. (1997) - Les épaves sarrasines d'Agay et de Cannes. Archeologia 337, p. 32-39.

JoNCHERAY, P. (1975) - Le navire de Bataiguier. Une épave du haut moyen-âge. Archeologia 85, p. 42-48.

Mc Pherson, K. (1993) - The Indian Ocean: A history of the People and the Sea, Oxford University Press.

Nascentes, A. (1932) - Dicionário Etimológico da Língua Portuguesa, 2 vols., Lisboa.

Pellegrini, G. B. (1978) - Terminologia marinara di origine araba in italiano e nelle lingue europee. In La navigazione mediterranea nell'alto medioevo (Settimane di Studio), vol. I-II (Spoleto centro di Studi sull'Alto Medioevo).

Picard, C. (1997) - L'Ocèan Atlantique musulman. De la conquète arabe à l'époque almohade. Navigation et mise en valeur des cotes d'al-Andalus et du Maghreb occidental (Portugal-Espagne-Maroc), Éditions de l'UNESCO, Paris.

Roxani, M. (2002) - Maritime trade in Medieval Aden, PhD Dissertation Princeton University.

Roxani, M. (2007) - Aden and the Indian Ocean Trade: 150 Years in the life of a Medieval Arabien Port, Chapel Hill, North Carolina, University of Carolina Press.

Tavares Carbonell Pico, M. A. (1963) - A terminologia naval portuguesa anterior a 1460, Sociedade de Língua Portuguesa, Lisboa.

Conimbriga, 53 (2014) 201-223 


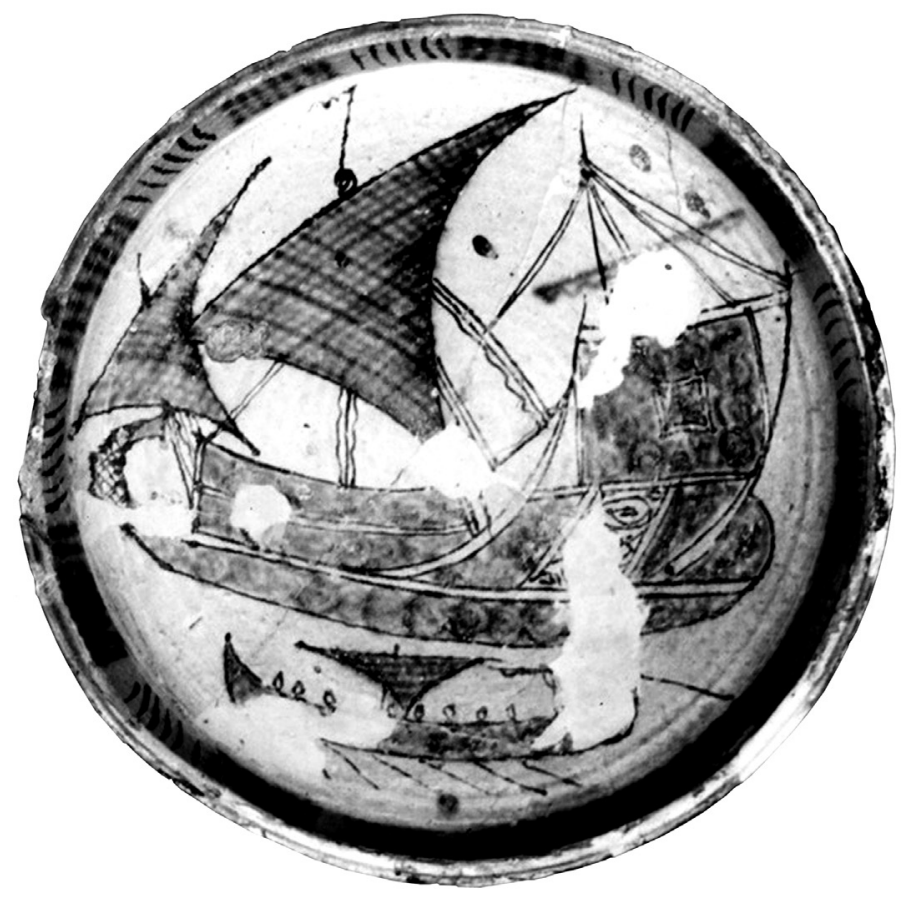

FIG. 1 - Bacino ceramico n.19 (Museo Nazionale di San Matteo, Pisa) 


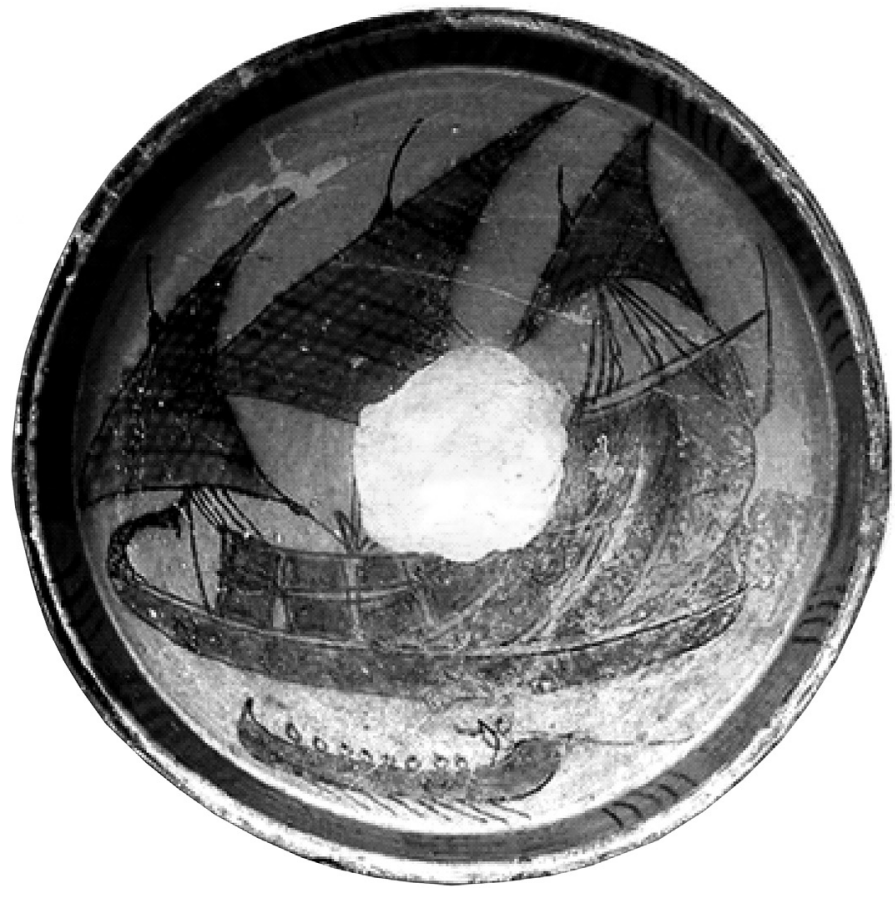

FIG. 2 - Bacino ceramico n.59 (Museo Nazionale di San Matteo, Pisa) 


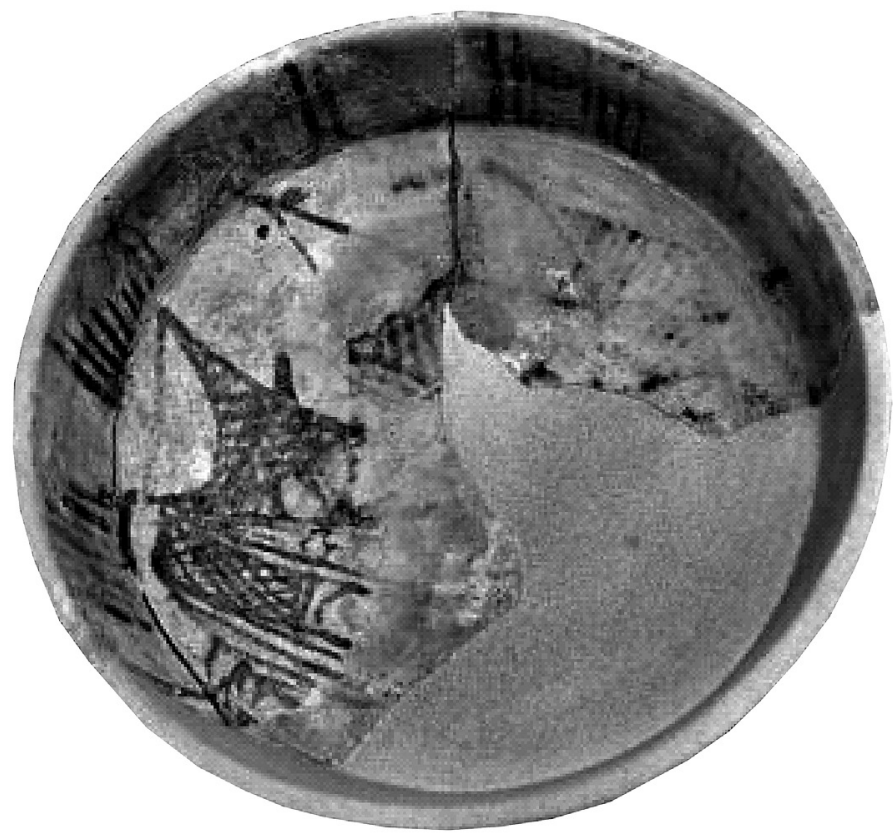

FIG. 3 - Ataifor di Cartagena (Museo ARQUA, Cartagena) 


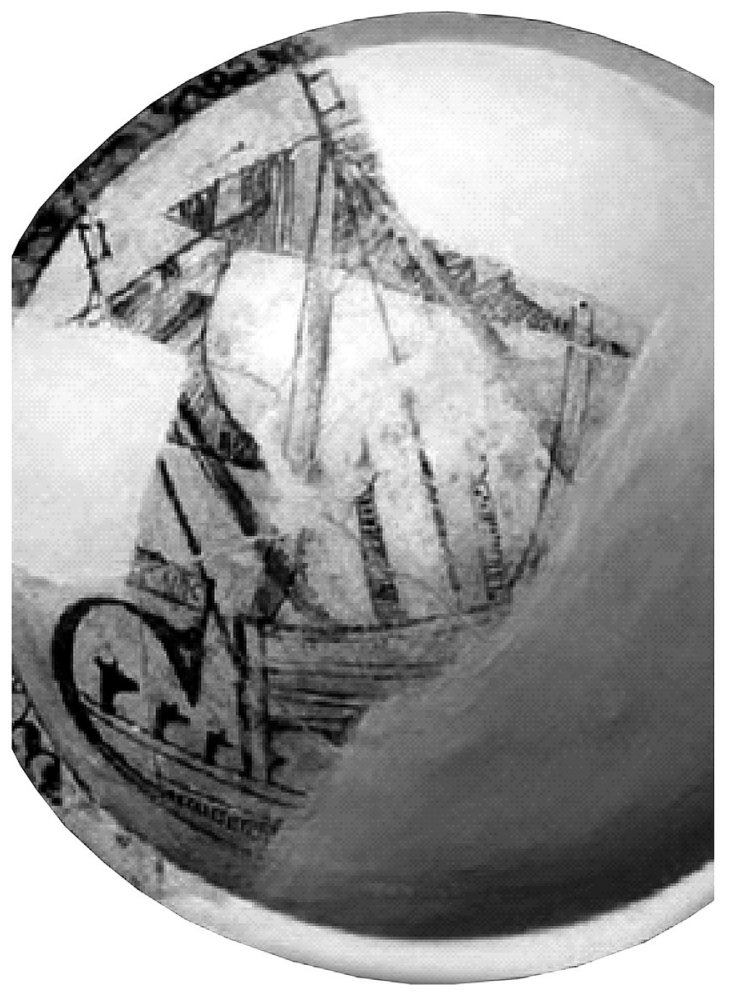

FIG. 4 - Ataifor di Dénia (MI Ayuntamiento-Museo Arqueológico, Dénia) 


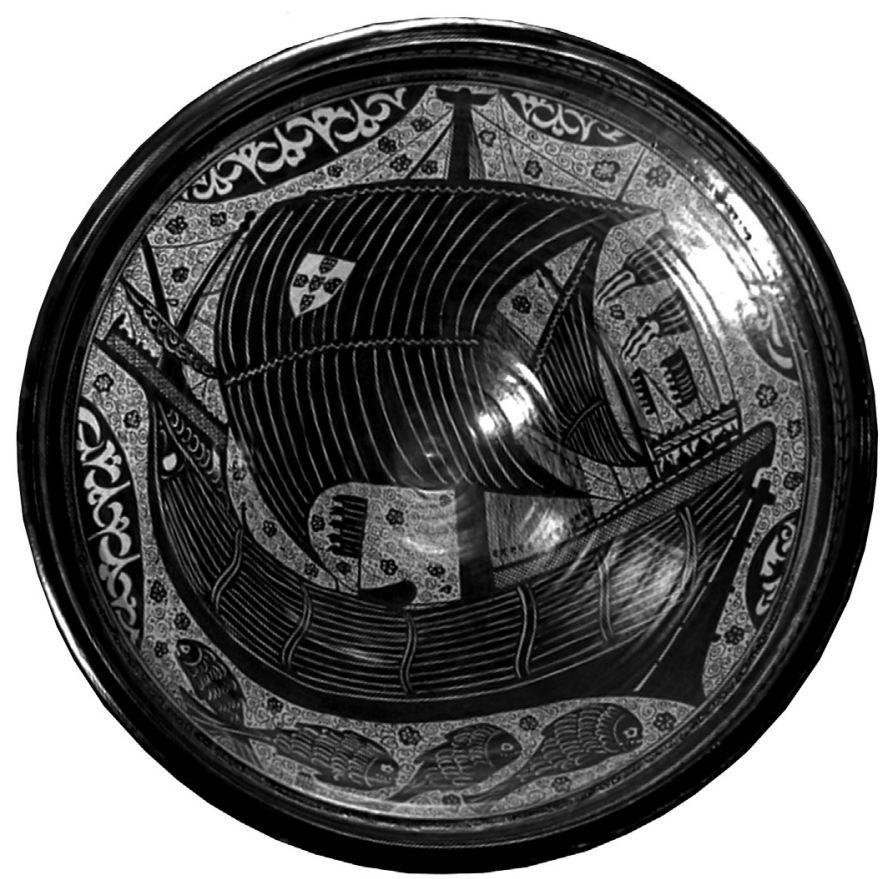

Fig. 5 - Bacino ceramico "Nave 1425-1450” (VAM Londra) 


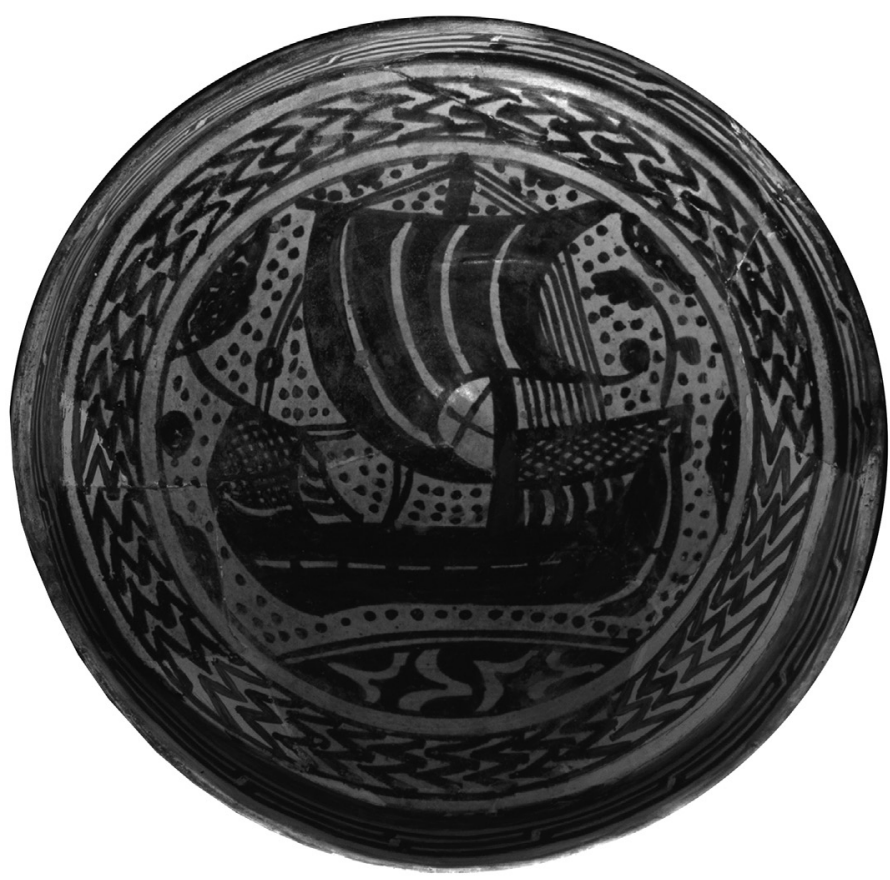

Fig. 6 - Piatto ceramico n.208 (Musée Sidi Qasim al-Jalizi, Tunisi) 


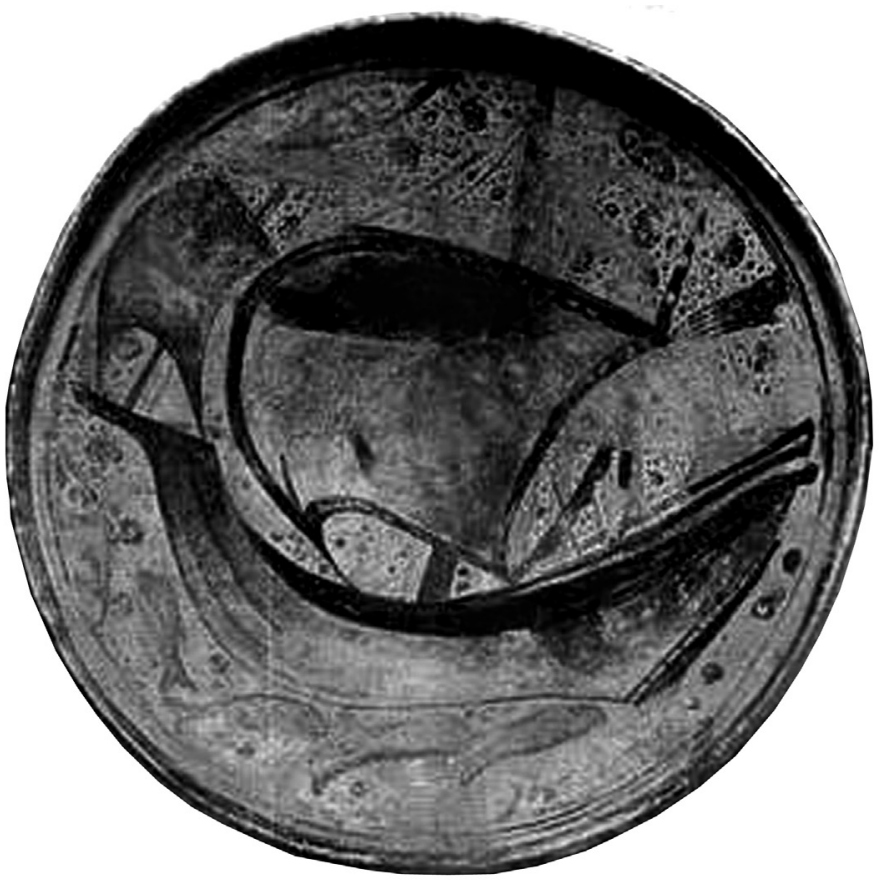

Fig. 7 - Piatto ceramico A/CE05724, XIV sec. (Museo Alcazaba de Málaga) 